\title{
The Structure and Properties of Electroceramics for Bone Graft Substitution
}

\author{
F.R. Baxter ${ }^{1,2, a}$, I.G. Turner ${ }^{1, b}$, C.R. Bowen ${ }^{1, c}$, J. P. Gittings $^{1}$, J.B. Chaudhuri ${ }^{2}$ \\ and R.W.C. Lewis ${ }^{1}$
}

${ }^{1}$ Centre for Orthopaedic Biomechanics, Department of Mechanical Engineering, University of Bath, Bath, BA2 7AY, United Kingdom

${ }^{2}$ Centre for Regenerative Medicine, Department of Chemical Engineering, University of Bath, Bath, BA2 7AY, United Kingdom

af.r.baxter@bath.ac.uk, bi.g.turner@bath.ac.uk, ${ }^{\mathrm{c}}$ c.r.bowen@bath.ac.uk

Keywords: Hydroxyapatite, Barium Titanate, Bone Graft, SEM, Piezoelectric

\begin{abstract}
Hydroxyapatite (HA) and barium titanate (BT) powders were mixed and sintered to form hydroxyapatite - barium titanate (HABT) ceramics. These materials were then poled and their piezoelectric properties were measured. The microstructure of unpoled samples was examined using scanning electron microscopy (SEM). The piezoelectric constants $\left(\mathrm{d}_{33}\right.$ and $\left.\mathrm{d}_{31}\right)$ of the ceramics were found to be dependent on the proportion of BT in the ceramic In materials containing less than $70 \% \mathrm{BT}$, no piezoelectric effect was found. Above this value, the piezoelectric constant increased with the addition of BT up to a value of $108 \mathrm{pCN}^{-1}$ for pure BT. Values of $\mathrm{d}_{33}$ for ceramics containing more than $80 \%$ BT are above values previously shown to have a positive influence on bone growth in vivo. SEM analysis indicated that the grain size within the materials decreased as the proportion of BT in the material was reduced. Examination of the microstructure of the ceramics indicated the presence of electrical domains in the $100 \%$ BT and $95 \%$ BT ceramics. Domains were not visible below 95\% BT. The reduction in grain size may influence the reduction in piezoelectric activity within the materials but cannot be considered to be the only cause.
\end{abstract}

\section{Introduction}

Stress generated potentials exist in bone in the form of piezoelectricity [1] and streaming potentials [2]. It has been shown that bone growth is influenced by these electromechanical signals [2, 3]. This has led to the suggestion that piezoelectric materials may offer advantages over existing bone graft substitutes although there has been limited research in this area. Calcium phosphate based bone graft substitutes in the form of hydroxyapatite (HA) have wide application in orthopaedic and dental applications as both fillers and coatings and as tissue engineering scaffolds [4]. Feng et al [5] examined the biocompatibility of hydroxyapatite - barium titanate (HABT) composites in canine subjects in order to exploit the known biocompatibility of HA and the piezoelectric nature of BT. They found that the composites appeared to promote osteogenesis in canine jawbones. However, there is a need for a better understanding of these materials if they are to be developed for clinical applications. The purpose of this study is to examine the piezoelectric properties and microstructural characteristics of a range of HABT ceramics with varying compositions.

\section{Materials and Methods}

The calcium phosphate powder used in this study was manufactured by Thermphos BV (Oldbury, UK). The BT powder was type Terro P, manufactured by Ferro (Haverhill, UK). The powders were mixed in a range of ratios to produce ceramics containing $0,20,40,60,70,75,80,85,90,95$, and 
$100 \%$ BT by volume. A volume gauge was used to measure out $0.353 \mathrm{~cm}^{3}$ powder for each individual tablet. Tablets were cold pressed in a $15 \mathrm{~mm}$ die at $60 \mathrm{MPa}$ for 10 seconds. They were then sintered at $1300^{\circ} \mathrm{C}$ for 2.5 hours with a heating and cooling rate of $60^{\circ} \mathrm{C} / \mathrm{hr}$. Post sintering, tablets measured $13 \mathrm{~mm}$ in diameter and $3 \mathrm{~mm}$ in thickness.

For the measurement of piezoelectric properties, tablets were poled using corona poling. The discs were heated to $130^{\circ} \mathrm{C}$ and exposed to a potential of $25 \mathrm{kV}$ at a point source height of $55 \mathrm{~mm}$ as they cooled to room temperature. The longitudinal and transverse piezoelectric constants $\left(\mathrm{d}_{33}\right.$ and $\mathrm{d}_{31}$ ) of the tablets were measured 24 hours after poling using a Take Control Piezometer PM25. Unpoled discs were sectioned vertically and the exposed faces were polished, followed by chemical etching using 10\% hydrofluoric acid. A Jeol JSM6310 scanning electron microscopy (SEM) operating with an accelerating voltage of $20 \mathrm{kV}$ was used to examine the microstructure of the materials.

\section{Results and Discussion}

Measurement of the piezoelectric properties revealed that the magnitudes of the constants $\left(\mathrm{d}_{33}\right.$ and $\mathrm{d}_{31}$ ) were directly related to the \% BT in the ceramic, as shown in Fig.1. Increasing the HA content leads to a rapid decrease in the magnitude of both constants. No piezoelectric effect was found in materials containing less than $70 \% \mathrm{BT}$.

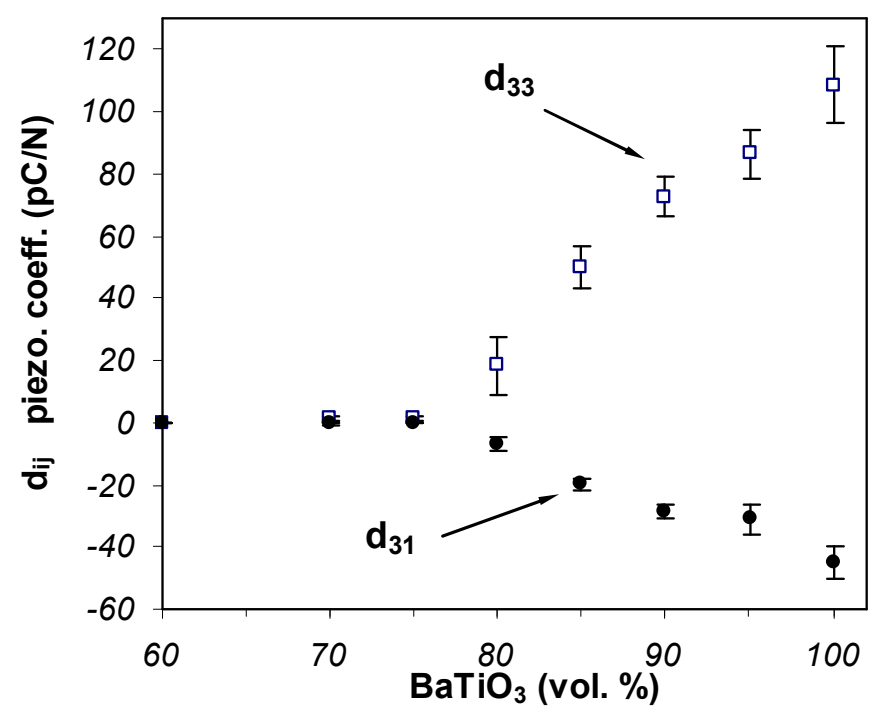

Fig.1 Variation of piezoelectric constants with \% BT. Error bars show standard deviation $(\mathrm{n}=6)$

Although the HABT ceramics produced in this study containing 70 and $75 \% \mathrm{BT}$ have piezoelectric constants below $1 \mathrm{pCN}^{-1}$, those containing $80 \%$ BT have a $\mathrm{d}_{33}$ of $18 \mathrm{pCN}^{-1}$. An earlier study by Feng et al [5] found bone growth in vivo to be influenced by piezoelectric ceramics with a $\mathrm{d}_{33}$ of $6 \mathrm{pCN}^{-1}$. Similarly, Calegari and Belangero [6] found improved bone growth in piezoelectric polymer tubes implanted in rat femurs when compared to non-polarised tubes. It may therefore be anticipated that HABT ceramics containing $80 \%$ or more BT will be capable of inducing a positive bone growth response.

SEM analysis was carried out on a range of samples. Selected images are shown in Fig.2. The SEM micrograph of the pure HA (Fig.2a) shows a fine grained polycrystalline HA structure. In comparison, the microstructure of pure BT (Fig.2b) contains large grains $(30-50 \mu \mathrm{m})$ in which striated ferroelectric domains are clearly visible. Ferroelectric domains are also visible, though less clearly, in the 95\% BT ceramic (Fig.2c). At 90\% BT ferroelectric domains are no longer apparent (Fig.2d). The presence of ferroelectricity in this material is, however, proven by the results found for the piezoelectric constant, which shows a relatively large $\mathrm{d}_{33}\left(72 \mathrm{pCN}^{-1}\right)$ at $90 \% \mathrm{HA}$. 
Grains shown in Fig.2b (pure BT) are 30-50 $\mu \mathrm{m}$ in size, while in Fig.2c (95\% BT) grains measure around $10 \mu \mathrm{m}$. At $90 \% \mathrm{BT}$, grain sizes are reduced to $1-2 \mu \mathrm{m}$. In other compositions, grain size decreases as the proportion of BT in the ceramic is reduced to zero.

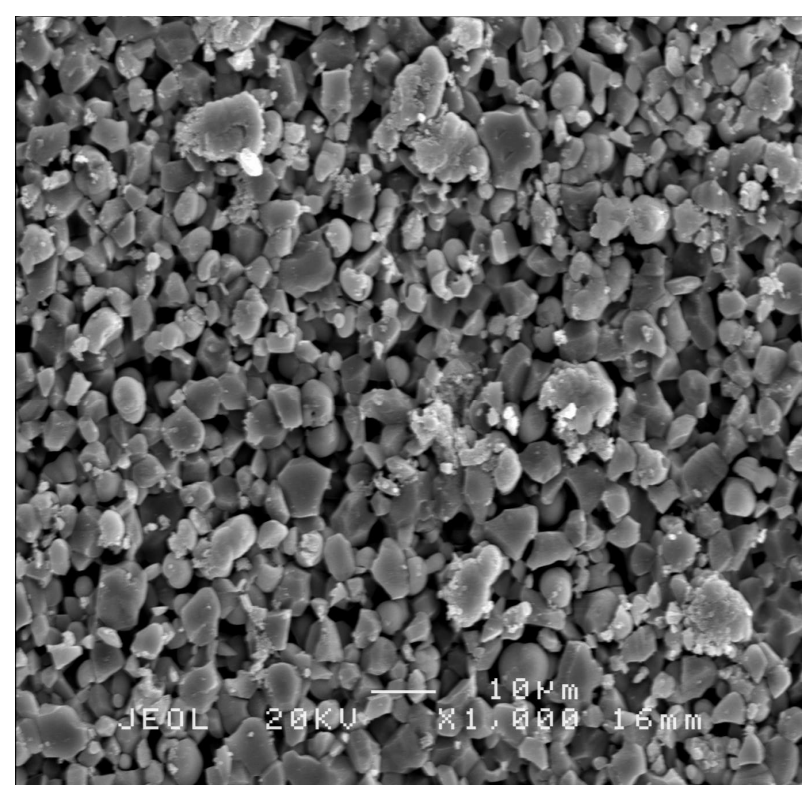

a) $100 \%$ HA (0\% BT)

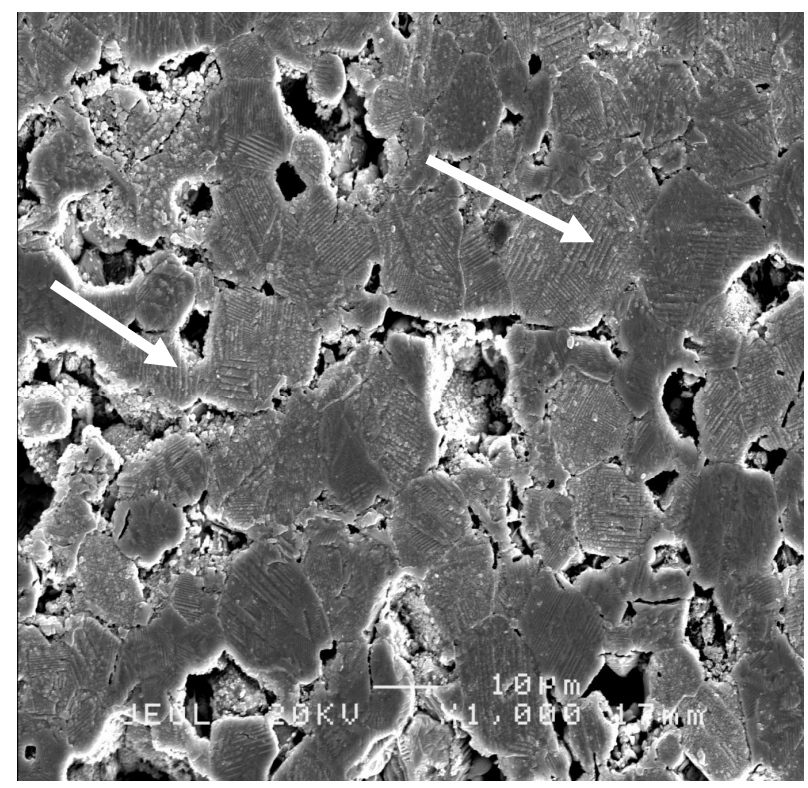

c) $95 \%$ BT

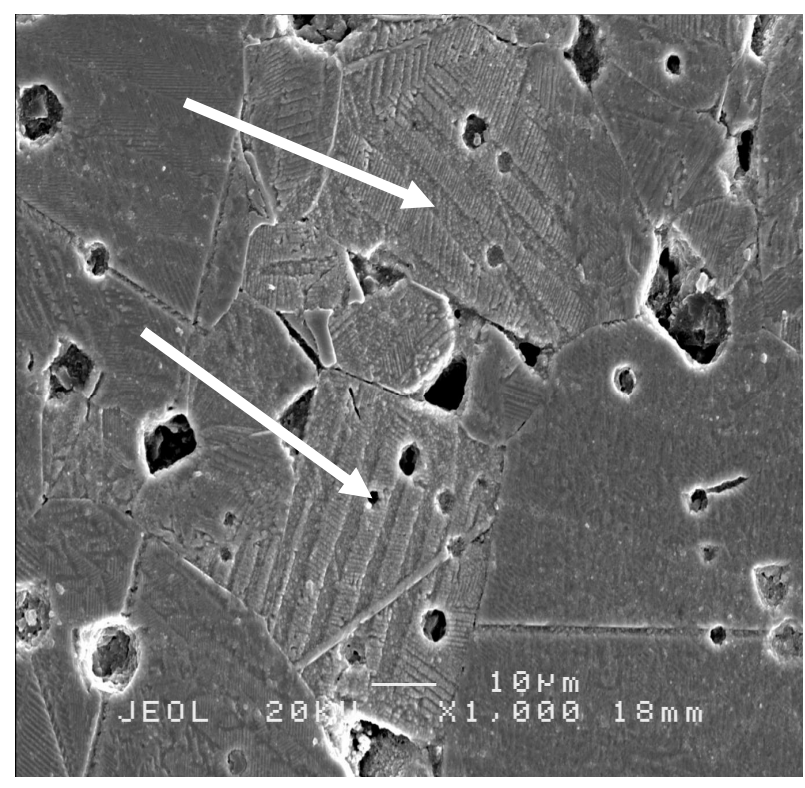

b) $100 \% \mathrm{BT}$

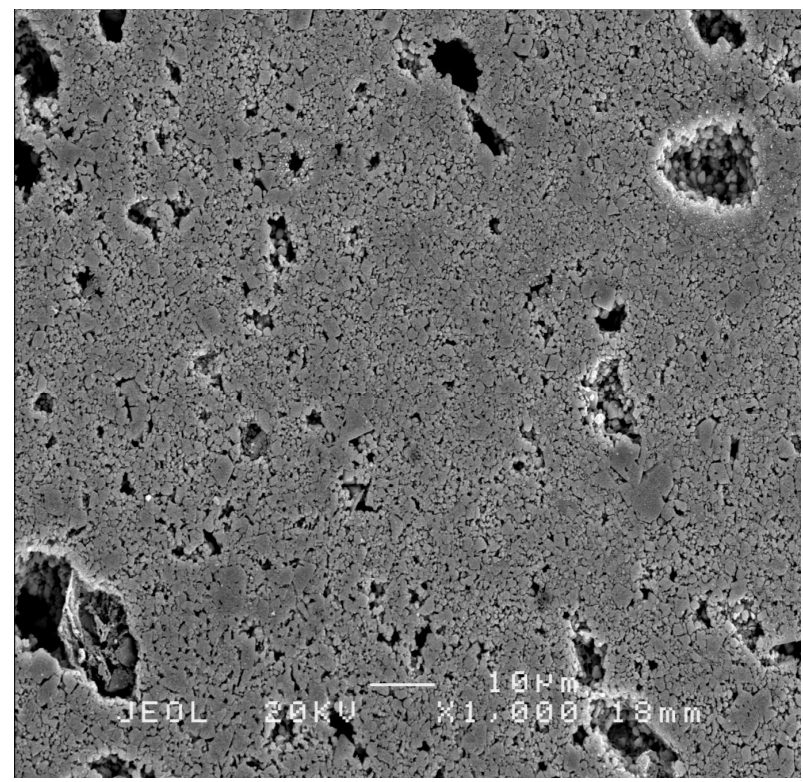

$90 \% \mathrm{BT}$

Fig.1. SEM micrographs of HABT ceramics. Original magnification x1000. Arrows show ferroelectric domains as indicated by visible striations. Scale bars $10 \mu \mathrm{m}$.

It appears, therefore, that the reduction in grain size associated with the increase in HA content contributes to the reduction in the piezoelectric constants. This suggestion is supported by the results of an earlier study [7] which found a rapid drop off in $\mathrm{d}_{33}$ in lead zirconate-titanate ceramics for grain sizes below $1 \mu \mathrm{m}$. Cau and Randall [8] found that, in fine grained materials, grain size affects domain wall motion and therefore influences piezoelectric properties. However, though Arlt [9] showed that the domain size in BT is related to the grain size, the link between domain size, grain size and piezoelectric properties in BT ceramics remains unclear for large grain sizes. For 
example, Takahasha et al [10] found high piezoelectric constants $\left(\mathrm{d}_{33}=350 \mathrm{pCN}^{-1}\right)$ in $\mathrm{BT}$ ceramics with grains measuring $2.1 \mu \mathrm{m}$, with no indication that a reduction in grain size leads to a lower $\mathrm{d}_{33}$ for grains between 1 and $100 \mu \mathrm{m}$ across. The reduction in piezoelectric properties of the materials examined in the current study cannot, therefore, be attributed to diminishing grain size alone. Further analysis of the composition of the sintered materials is therefore necessary to gain a fuller understanding, including analysis of possible reactions between HA and BT.

\section{Conclusions}

HABT ceramics with a range of piezoelectric properties have been produced. The piezoelectric constant $\left(\mathrm{d}_{33}\right)$ of these materials varies from $0 \mathrm{pCN}^{-1}$ at $70 \%$ BT to $108 \mathrm{pCN}^{-1}$ at $100 \%$ BT. The $\mathrm{d}_{33}$ of the ceramics containing over $80 \% \mathrm{BT}$ is in a range that may be expected to influence bone growth in vivo. SEM micrographs show that grain size decreased as the proportion of BT in the ceramic is reduced. Electrical domains were clearly visible in ceramics containing $100 \%$ and $95 \%$ BT. The reduction in grain size in these materials may contribute to, but cannot explain completely, the reduction in the piezoelectric constants of the HABT as the HA content increases.

Further investigation of the composition of these materials after sintering and of the influence of the microstructure and piezoelectric properties of the ceramics on cell responses in vitro is planned.

\section{Acknowledgements}

The authors would like to thank the EPSRC for their funding of this work and the Centre for Electron Optical Studies at the University of Bath for the use of their facilities.

\section{References}

[1] E. Fukada and I. Yasuda: Journal of the Physical Society of Japan Vol. 12(10) (1957), p. 1158.

[2] S.C. Cowin and M.L. Moss in Bone Mechanics Handbook, edited by S.C. Cowin CRC Press, Boca Raton, FL (2001).

[3] G.W. Hastings and F.A. Mahmud: Journal of Biomedical Engineering Vol. 10 (1988), p. 515

[4] C.T. Laurencin: Bone Graft Substitutes (ATSM International, West Conshohocken, PA, 2003).

[5] J.Q. Feng, H.P. Yuan and X.D. Zhang: Biomaterials Vol. 18(23) (1997), p. 1531.

[6] B. Callegari and W.D. Ballengero: Acta Orthopaedica Brasileira Vol. 12 (2004), p. 160.

[7] W. Hackenberger, M.J. Pan, V. Vedula, P. Pertsch, W.W. Cao, C.Randall and T. Shrout (1998), SPIE, 3324: 28-.36.

[8] W. Cau and C.A. Randall: Journal of Physics and Chemistry of Solids Vol. 57(10) (1996), p. 1499-1505.

[9] G. Arlt: Journal of Materials Science Vol. 25 (1990) p. 2655.

[10] H. Takahashi, Y. Numamoto, J. Tani and S. Tsurekawa: Japanese Journal of Applied Physics Vol. 45(9B): p.7405. 